\title{
Sterben Controllerkarrieren aus?
}

Die Controller könnten aktuell sehr zufrieden sein. Sie haben viel spannendere Aufgaben als früher, genießen ein besseres Standing beim Management, sind zu deren Mitspielern aufgestiegen (Business Partner) und bringen es mittlerweile in die Top-Etagen selbst von Großunternehmen. Controller sind vorstandsfähig geworden. Warum sollte man also unruhig werden und Gefahren wittern?

Es gibt sie aber tatsächlich, virulente aktuelle Probleme und Bedrohungen, denen Controller ausgesetzt sind. Eines davon ist der demografische Effekt, der auch nicht am Controlling vorbeigeht. Qualifizierten Führungsnachwuchs zu bekommen, wird immer schwieriger. Der "War for Talents" ist in vollem Gange. Zugleich sind mit dem angesprochenen inhaltlichen Wandel („vom Erbsenzähler zum Business Partner“) erhebliche Veränderungen der Anforderungen an Controller verbunden. Die geforderten Eingangsqualifikationen unterscheiden sich nicht von denen anderen ambitionierten Führungsnachwuchses. Gefordert wird nicht der Zahlen- und Methodenspezialist, sondern der betriebswirtschaftliche Generalist, der außerdem mit den Controlling-Instrumenten sicher umgehen kann. Zugleich sind die Erwartungen erheblich gestiegen, die den Einsatz der Controller betreffen. Das Controlling gilt in vielen Unternehmen als der Bereich, in dem man am meisten arbeiten muss.

Bei der Gewinnung von Controlling-Nachwuchs geht es also um die besten Studenten, die dafür begeistert werden müssen, ihre Spezialisierungen in das Controlling oder in controllingnahe Gebiete zu legen. Allerdings ist die Konkurrenz gerade bei einem solchen Profil sehr hoch. Wenn man alles zusammennimmt, muss im selben Teich gefischt werden, aus dem auch die Berater geangelt werden. Diese haben aber zumindest ein deutlich besseres Einkommen vor Augen.

Nachteile bestehen auch hinsichtlich des Images des Controllings an den Hochschulen. Wer in einem Praktikum im Controlling-Bereich arbeitet, hat es zumeist mit wenig spannenden, transaktionsnahen Routinetätigkeiten zu tun. Das ist nicht gerade hilfreich, um Spitzenleute für das Controlling zu begeistern. Auch die Ausbildung selbst scheint nicht so anziehend zu sein, dass die Attraktivität des Faches innerhalb der Leistungsspitze der Studierenden hoch ist. Wie wir aus

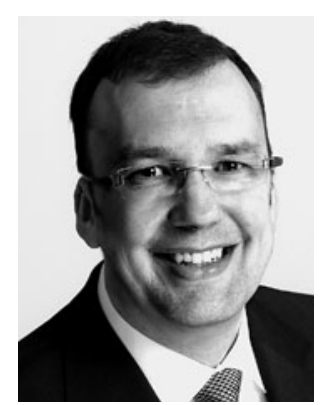

Utz Schäffer

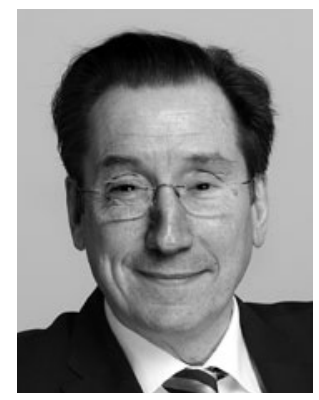

Jürgen Weber

einer am Institut durchgeführten empirischen Studie an vielen Hochschulen wissen, werden die zukünftige Arbeitstätigkeit und das Image des Controllerberufes von den Studierenden relativ schlecht bewertet. Auch wir an der WHU haben es noch nicht geschafft, Controlling bei den Studierenden auf das Attraktivitätsniveau von Investmentbanking oder Beratung zu heben. Aber auch Marketing und Innovationsmanagement sind für viele Studierende spannender als das etwas trocken erscheinende Fach Controlling.

Was ist also zu tun? Unserer Meinung nach liegt ein wesentlicher Hebel in der Revitalisierung des etwas in Vergessenheit geratenen Themas „Goldfischteich für das Management“, der für Controlling-Nachwuchs aufzubauen und entsprechend zu kommunizieren ist. Wir müssen klarmachen, dass Controller mit ihrer Leidenschaft für Wirtschaftlichkeit sowie ihrer Fähigkeit, unterschiedliche Interessen in einem gemeinsamen Handeln zu bündeln, die besten Voraussetzungen haben, 
leitende Managementfunktionen zu übernehmen bzw. genau das in ihrer Zeit als Controller sehr gut lernen können. Eine solche Karrieregestaltung hilft nicht nur dem einzelnen Controller, sondern auch und in erster Linie dem Unternehmen: Es reicht in der heutigen Zeit nicht mehr, dass man sich für die Spezialisten in „calculative practices“ hält, die entsprechende Aufgaben für das Management übernehmen. Die betriebswirtschaftlichen Techniken müssen vielmehr das Einmaleins aller Führungskräfte sein. Nur dann können Unternehmen heute erfolgreich agieren.

Nachwuchsarbeit im Controlling hat also sehr viel mit der perspektivischen Entwicklung des Controllings zu tun.
Spätestens seit dem Ruf nach dem Business Partner brauchen wir im Controlling Nachwuchskräfte, die - pointiert gesagt nicht das Ziel haben, bei ihrer Pensionierung auf dem Stuhl des obersten Unternehmenscontrollers zu sitzen, sondern auf dem des CEO. Hierauf muss sowohl die Personalakquisition als auch die folgende Personalentwicklung im Unternehmen ausgerichtet sein. Wenn die Beispiele von Koch (CEO METRO), Bock (CEO BASF) oder Ginthum (Vetriebsvorstand BSH) dann Schule machen, brauchen wir uns um den Controller-Nachwuchs keine großen Sorgen mehr zu machen, auch wenn es die traditionellen Controller-Karrieren in $\mathrm{Zu}$ kunft nicht mehr geben wird.

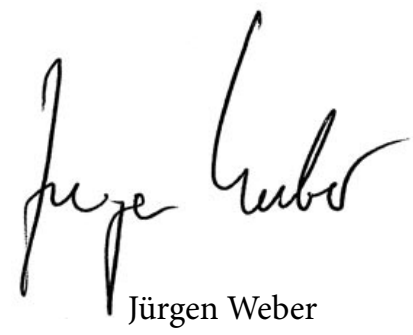

